\title{
Luteolin reduces cancer-induced skeletal and cardiac muscle atrophy in a Lewis lung cancer mouse model
}

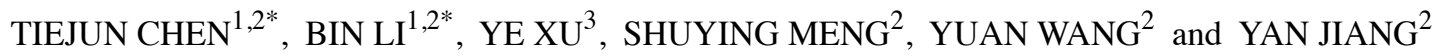 \\ ${ }^{1}$ Department of Medical Oncology, ${ }^{2}$ Translational Medical Laboratory, Benxi Central Hospital, Benxi, Liaoning; \\ ${ }^{3}$ Geriatric Department, General Hospital of Benxi Iron and Steel Co., Ltd., Benxi, Liaoning 117000, P.R. China
}

Received November 14, 2017; Accepted May 15, 2018

DOI: $10.3892 /$ or.2018.6453

\begin{abstract}
Luteolin was recently demonstrated to suppress tumor growth by interfering with nuclear factor (NF)- $\mathrm{kB}$ activation. As the NF- $\kappa B$ pathway plays a critical role in muscular atrophy associated with cancer cachexia, we aimed to investigate the potential of luteolin to alleviate cancer-associated cachexia in a Lewis lung cancer mouse model. C57BL/6 mice were divided into three groups: A control group, a model group and a luteolin group. Mice in the model and luteolin groups received a subcutaneous injection of Lewis lung cancer cells, while the control group received PBS. Subsequently, the tumor mass, serum, gastrocnemius muscle and heart were collected on day 21. The serum, gastrocnemius muscle and heart were weighed and prepared for use in enzyme-linked immunosorbent assay (ELISA), western blotting (WB) and quantitative reverse transcription polymerase chain reaction (qRT-PCR) analyses. The results revealed that the tumor-free body mass was significantly reduced in tumor-bearing mice compared with that of mice in the other groups. The gastrocnemius muscle mass and heart mass were greater in the luteolin treatment group than in the control group. Tumor necrosis factor (TNF)- $\alpha$ and interleukin (IL)-6 levels were lower in the luteolin treatment group than in the model group. In addition, according to the results of the WB and qRT-PCR analyses, the expression of the E3 ubiquitin ligase muscle RING finger-containing protein 1 (MuRF1) was downregulated in skeletal muscle and cardiac muscle, whereas atrogin-1 was downregulated only in skeletal muscle in the luteolin treatment group vs. the model group. Furthermore, IкB kinase $\beta$ (IKK $\beta$ ) and phospho-p65 were significantly downregulated in skeletal muscle and cardiac tissue, whereas the expression of p-p38 was downregulated
\end{abstract}

Correspondence to: Dr Bin Li, Department of Medical Oncology, Benxi Central Hospital, 29 Shengli Road, Benxi, Liaoning 117000, P.R. China

E-mail: libin781001@126.com

*Contributed equally

Key words: cancer cachexia, muscle atrophy, luteolin, NF-кB pathway only in skeletal muscle in the luteolin treatment group when compared with their expression levels in the model group, as determined by the WB analysis. In conclusion, from the current results, we conclude that luteolin is able to reduce inflammatory burden, downregulate the expression of genes associated with muscle protein breakdown, and protect skeletal and heart muscle from cancer-induced wasting and loss in vivo. Therefore, luteolin has the potential to be developed into a novel anti-cachetic agent.

\section{Introduction}

Cancer cachexia is a complex syndrome that is characterized by an ongoing loss of skeletal muscle mass (1). If a decrease in fat mass occurs, conventional nutritional support is insufficient to overcome the loss. Cachexia induced by malignant diseases can lead to severe consequences, including poor prognosis, diminished quality of life, and decreased radiochemotherapy efficacy (2-4). Cachexia occurs in up to $80 \%$ of patients with advanced stage cancer, and $\sim 50 \%$ of patients have cachexia at the time of initial cancer diagnosis. Furthermore, cachexia is responsible for more than $20 \%$ of cancer-associated deaths (2). For these reasons, there is increasing focus on cancer cachexia in the field of cancer therapy.

Currently, the underlying mechanisms of cachexia remain largely unknown; correspondingly, therapeutic progress is limited (5). Skeletal muscle loss is the prominent characteristic of cachexia; however, at present, there are no effective standard measures of cancer cachexia as it is a complex clinical syndrome with unclear underlying mechanisms. Therefore, the focus of treatment is on the inhibition of skeletal muscle loss to mitigate the associated adverse affects.

The molecular mechanisms of skeletal muscle atrophy/hypertrophy are intricate and remain poorly defined; however, an opportunity for downstream intervention has been identified that may circumvent the variations and redundancy in upstream mediators, and these findings may ultimately translate into new targeted therapies. Specifically, a number of studies (6-8) have indicated that two signaling mediators are required to upregulate the expression of the key E3 ligases, muscle RING finger-containing protein 1 (MuRF1) and muscle atrophy $\mathrm{F}$ box protein (MAFbx, otherwise known as atrogin-1), which predominantly mediate sarcomeric breakdown during muscle loss. Tumor necrosis factor-like weak inducer of 
apoptosis (TWEAK) and, in particular, tumor necrosis factor (TNF)- $\alpha$ induce MuRF1 upregulation via NF- $\kappa B$, resulting in the degradation of myosin heavy chains (MyHC) (9).

The transcription factor NF- $\kappa \mathrm{B}$ was first identified over 20 years ago and is a regulator of the expression of the $\mathrm{kB}$ light chain in B cells. A study by Rhoads et al (10) showed that there was a $25 \%$ increase in 655 phosphorylation and a significant decrease in the expression of $\mathrm{I \kappa} B \alpha$ in gastric cancer patients when compared with the levels in controls, which suggest that the activation of the classical NF- $\mathrm{KB}$ pathway in the muscle tissue of patients accompanies cancer cachexia. Additionally, a report by Wysong et al (11) suggested that NF- $\mathrm{\kappa B}$ inhibition via the I $\kappa \mathrm{B}$ complex is able to protect against cancer-associated cardiac atrophy. According to these findings, it seems feasible that skeletal muscle loss and cardiac atrophy could be inhibited by disrupting the NF- $\mathrm{kB}$ pathway.

Compounds of natural origin can be used as new and innovative therapeutic agents for the treatment of diseases. Luteolin (3-5,7-tetrahydroxy flavone) is a natural flavonoid present in several plants. A number of studies have reported its potential anticancer activity and its inhibition of NF- $\mathrm{kB}$ activation in cancer; moreover, suppression of NF- $\mathrm{KB}$ by luteolin can activate TNF- $\alpha$-induced apoptosis (12). Luteolin decreases $\mathrm{NF}-\kappa \mathrm{B}$ activation at both the transcriptional and translational levels and inhibits the production of the inflammatory mediators interleukin (IL)-6, IL-8, and vascular endothelial growth factor (VEGF) by TNF-triggered human keratinocytes (13).

However, to the best of our knowledge, there are no reports indicating that luteolin can inhibit skeletal and cardiac muscle loss. Therefore, in the present study, we investigated whether luteolin inhibits cancer-induced skeletal muscle and cardiac atrophy by inhibiting the NF-kB pathway in vivo. Our results suggest that luteolin is a promising candidate to be developed into an effective therapeutic agent for the treatment of muscle loss associated with cancer cachexia.

\section{Materials and methods}

Cell culture and natural drug preparation. Lewis lung cancer (LLW) cells were purchased from Shanghai Institutes for Biological Sciences (Shanghai, China) and were grown in Dulbecco's modified Eagle's medium (DMEM) (Biosera, Kansas City, MO, USA) supplemented with $10 \%$ fetal bovine serum (FBS), $100 \mathrm{U} / \mathrm{ml}$ penicillin, and $100 \mu \mathrm{g} / \mathrm{ml}$ streptomycin, in a humidified incubator at $37^{\circ} \mathrm{C}$ with $5 \% \mathrm{CO}_{2}$.

Luteolin was purchased from Nantong Feiyu Biological Technology Co., Ltd. (>98\%; FY14650427) and diluted in PBS for use in the present study. The structure of luteolin is shown in Fig. 1.

In vivo model of cancer cachexia. Cachexia induced by LLC is frequently used (14-16) as a preclinical and experimental model as it resembles clinical cachexia, including the resulting physiological and metabolic characteristics; hence, this in vivo model has been used for many years and is an established model of cancer-induced cachexia. Mice were obtained from Shanghai S\&P-Shall Kay Laboratory Animal Co.,Ltd. (specific pathogen-free certificate numbers: SCXK Hu 2008-0016). The 30 male 4-to 6-week-old (18-22 g) C57BL/6 mice obtained were housed in a uniform temperature room $\left(26^{\circ} \mathrm{C}\right)$ under a 12-h light/dark cycle (light from 08:00 to 20:00). They were placed in closed cages and given free access to chow and water. The feed used and protocols carried out in this study were in accordance with the regulations of the Institutional Animal Care and Use Committee (IACUC) of Jiangsu University (Jiangsu, China). The experimental mice were randomly divided into the following three groups (10 mice per group) after 1 week of acclimation: A control group, a model group, and a luteolin group. On the first day of the experiment, $200 \mu \mathrm{l}$ of PBS was injected subcutaneously into the right flank of each mouse in the control group. Mice in the other two groups were each injected subcutaneously at the same position with $\sim 1 \times 10^{7}$ LLW cells in $200 \mu \mathrm{l}$ of PBS. From day 7 to 24 , mice in the treatment group were treated with luteolin. Luteolin was diluted in PBS and delivered via intragastric administration $(20 \mathrm{mg} / \mathrm{kg} / \mathrm{day}, 0.3 \mathrm{ml})$. The control and model groups received daily sham treatments of PBS alone $(0.3 \mathrm{ml})$. Body weight was measured for each group using an electronic scale at the beginning and end of the experiment, and tumor-free body weight was estimated by subtracting the weight of the excised tumor. On day 24, blood samples were obtained from the orbital vein, centrifuged at $3,000 \mathrm{rpm}$ at $4^{\circ} \mathrm{C}$ for $20 \mathrm{~min}$ within $1 \mathrm{~h}$, and then stored at $-80^{\circ} \mathrm{C}$. Immediately after blood withdrawal, the mice were sacrificed by cervical dislocation. The tumors, hearts, bilateral gastrocnemius muscles and bilateral tibialis anterior muscles were immediately harvested and weighed. The muscle tissues were quickly frozen in liquid nitrogen and stored at $-80^{\circ} \mathrm{C}$.

Detection of cytokines. Using commercially available enzyme-linked immunosorbent assay (ELISA) kits, the serum cytokines TNF- $\alpha$ and IL- 6 were detected. The detection kits contain pre-coated plates (mouse IL-6: DKW12-2060; mouse TNF- $\alpha$ : DKW12-2,720; Dakewei Biotech.Co.,Ltd., Shenzheng, China) and were used in accordance with the manufacturer's protocols. Serum from each animal $(50 \mu \mathrm{l})$ was assayed in duplicate. Standard curves were created using recombinant mouse IL- 6 and TNF- $\alpha$ to allow quantitative calibration.

Western blot (WB) analysis. After muscle and cardiac tissues were homogenized by adding a protein lysis buffer (Beyotime Institute of Biotechnology, Haimen, Jiangsu, China), the homogenates were centrifuged for $10 \mathrm{~min}$ at $12,000 \mathrm{rpm}$ at $4^{\circ} \mathrm{C}$, and the supernatant was aspirated. A BCA Protein Assay Kit (Thermo Fisher Scientific, Inc., Waltham, MA, USA) was used according to the manufacturer's protocol to determine the protein concentrations. The proteins were separated by gel electrophoresis (Bio-Rad Laboratories, Inc., Hercules, CA, USA) and transferred onto polyvinylidene fluoride (PVDF) membranes (Millipore Corp., Bedford, PA, USA). The PVDF membranes were incubated with the indicated primary antibodies overnight at $4^{\circ} \mathrm{C}$ with gentle agitation (anti-atrogin-1: cat. no. ab74023, anti-MuRF1: cat. no. ab172479, anti-phospho-p65: cat. no. ab86299, anti-IKK $\beta$ : cat. no. ab32135, anti-p38: cat. no. ab170099, anti-p-p38: cat. no. ab195049; Abcam, Cambridge, MA, USA) and with anti-NF-kB p65 (cat. no. 8242; Cell Signaling Technology, Inc., Danvers, MA, USA). The diultions for all antibodies was 1:1,000 except anti-atrogin-1 and anti-phospho-p65 with 1:500. Using an image scanning 


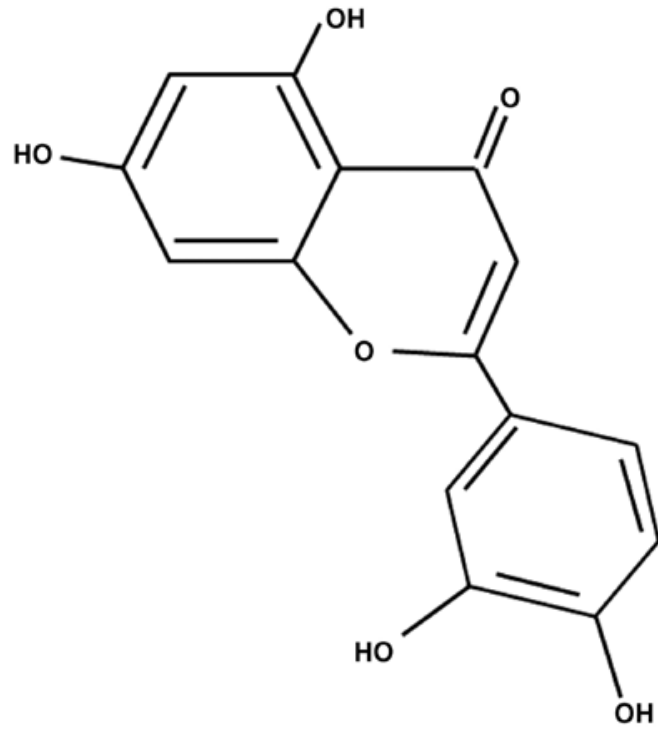

Figure 1. Chemical structure of luteolin $\left(\mathrm{C}_{15} \mathrm{H}_{10} \mathrm{O}_{6}\right)$.

densitometer connected to a chemiluminescence system, antibody binding was detected after incubation with a horseradish peroxidase-conjugated secondary antibody (Cell Signaling Technology, Inc.). Quantitative analysis of protein expression levels was performed using ImageJ software (National Institutes of Health, Bethesda, MD, USA). The protein expression levels were normalized to $\beta$-actin expression.

Real-time quantitative reverse transcription PCR ( $q R T-P C R)$ analysis. We measured the mRNA expression of MuRF1 and atrogin-1 in the gastrocnemius muscles and cardiac muscles of the three groups by qRT-PCR analysis, as described in detail elsewhere. In brief, total RNA was extracted from the whole gastrocnemius muscle and cardiac muscle using a RiboPure ${ }^{\mathrm{TM}}$ Kit (Life Technologies Japan Inc., Tokyo, Japan) according to the manufacturer's instructions. First-strand cDNA was generated by reverse transcription using a High-Capacity RNA-to-cDNA Kit (Takara Bio Inc., Beijing, China), and the resulting cDNA was stored at $-20^{\circ} \mathrm{C}$ for later analysis. qRT-PCR was performed using a TaqMan Fast Universal PCR Master Mix (Takara Bio Inc.) and a Thermal Cycler Dice RealTime System II (Takara Bio Inc.). The levels of mRNA were determined using the primers shown in Table I. The expression of the genes was normalized to the expression of $\beta$-actin, and the results are expressed as relative differences.

Statistical analysis. All data represent the means \pm standard deviation (SD), and ANOVA with Tukey's post hoc comparison was used to identify significant differences between the groups. This analysis was performed with SPSS version 18.0 (SPSS, Inc., Chicago, IL, USA), and a two-sided P-value $<0.05$ was considered to indicate statistical significance.

\section{Results}

Effect of luteolin on tumor-free body weight and weight of gastrocnemius muscles and heart. One of the main characteristics of cancer cachexia is the loss of skeletal muscle. On
Table I. List of primers used for PCR analysis.

Primer sequence (5'-3')

\begin{tabular}{ll}
\hline Atrogin-1 & F: TCCAGTGAGGAGCAGTTCAG \\
& R: CAGTGTGATTGGCATTTGGT \\
FuRF-1 & F:ACGAGAAGAAGAGCGAGCTG \\
& R: CAAAGTCAATGGCCCTCAAG \\
$\beta$-actin & F: TCAGTGCCGGCCTCGTCTCAT \\
& R: TGACCAGGCGGCCAATACGG
\end{tabular}

F, forward; R, reverse.

day 21 , the tumor-free body weights of tumor-bearing mice were significantly lower than those of the control and model mice. The masses of the heart muscle, gastrocnemius muscle, and tibialis muscle were obviously reduced in the model group compared with those of control mice; however, the masses were significantly increased in model mice treated with luteolin compared with those in the model group. Unfortunately, the masses in the luteolin group remained lower than those of normal mice (Fig. 2).

Effect of luteolin on TNF- $\alpha$ and IL-6. Cytokines, especially TNF- $\alpha$ and IL-6, are released at significantly increased levels by tumors, and trigger and promote the progression of cachexia $(8,17,18)$. In the present study, we first detected TNF- $\alpha$ and IL- 6 in the sera of mice using ELISAs. In the model mice, these two cytokines were obviously elevated compared with their levels in the control mice, while their levels were significantly reduced by luteolin treatment compared with those in the model mice. The level of TNF- $\alpha$ remained significantly higher in the luteolin-treated mice than that in the control mice (Fig. 3).

Effect of luteolin on atrogin-1 and MuRF-1 expression. MuRF1 and atrogin-1 have been identified as important markers of muscle degradation, and the upregulation of these proteins has been reported during the degradation of skeletal muscle protein $(6,19,20)$. Therefore, we detected the expression of MuRF1 and atrogin-1 at the protein and transcript levels by WB analysis and qRT-PCR, respectively, in gastrocnemius muscle samples. The protein levels of MuRF1 and atrogin-1 were obviously upregulated in the model mice; however, this upregulation was attenuated by luteolin treatment (Fig. 4A and B). Correspondingly, the mRNA levels of MuRF1 and atrogin-1 were significantly elevated in the model mice, and this elevation was inhibited by luteolin treatment in the skeletal muscle (Fig. 4C). In addition, we detected MuRF1 and atrogin-1 expression in cardiac muscle to assess their correlation with the reduction of heart mass. The levels of MuRF1 and atrogin-1 were obviously upregulated in the model mice, but only the increase in MuRF1 was significantly inhibited by luteolin treatment (Fig. 4A-C).

Effect ofluteolinon $N F-\kappa B$ signaling andp38mitogen-activated protein kinase $(M A P K)$. In view of the established critical 
Heart weight

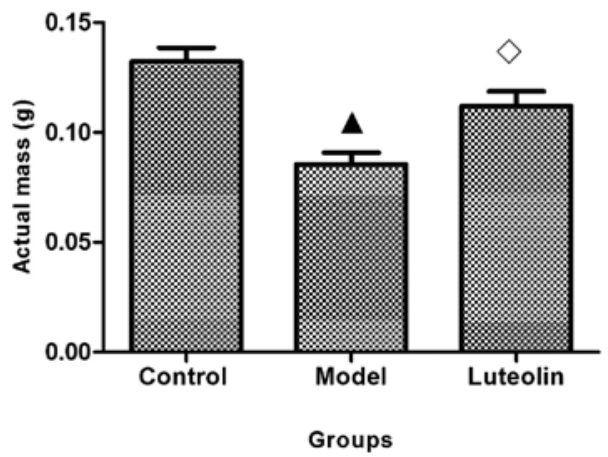

Tibialis weight

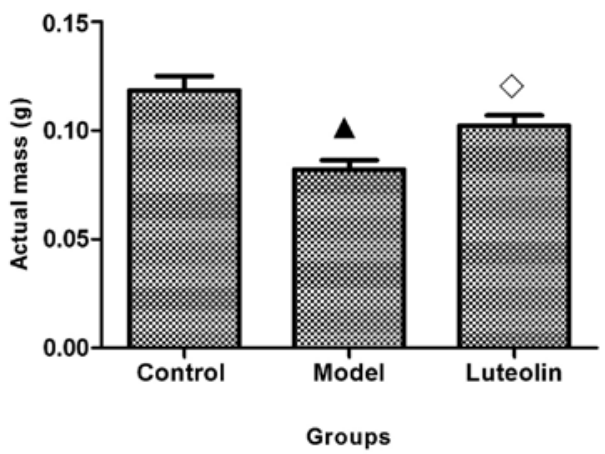

Gastrocnemius weight

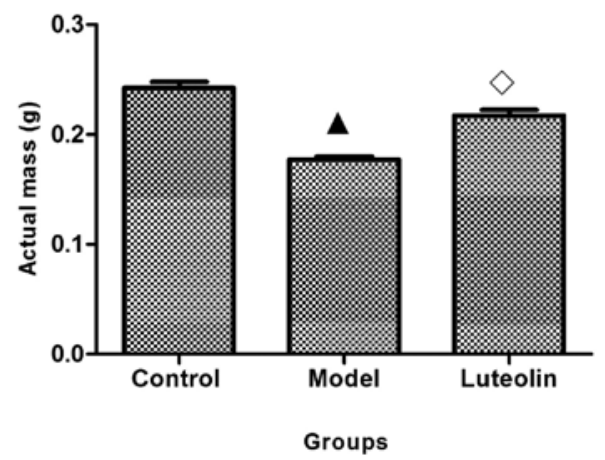

Tumor-free body weight

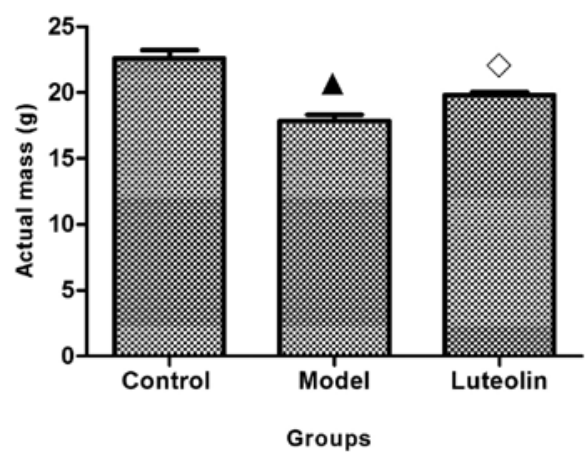

Figure 2. Effects of luteolin on the mass of the heart, gastrocnemius muscle and tibialis anterior muscle, and on tumor-free weight. All mice were divided into a control group, a model group, and a luteolin group (10 mice/group). At the end of the experiment, the heart, gastrocnemius muscle, tibialis anterior muscle, and tumor mass were collected. Tumor-free weight was determined as 'total weight-tumor weight'. Each bar represents the mean \pm SD. Statistical significance vs. the control group $\left({ }^{\boldsymbol{\Delta}} \mathrm{P}<0.05\right)$; statistical significance vs. the model group $\left({ }^{\diamond} \mathrm{P}<0.05\right)$.

\section{Serum IL-6 level}

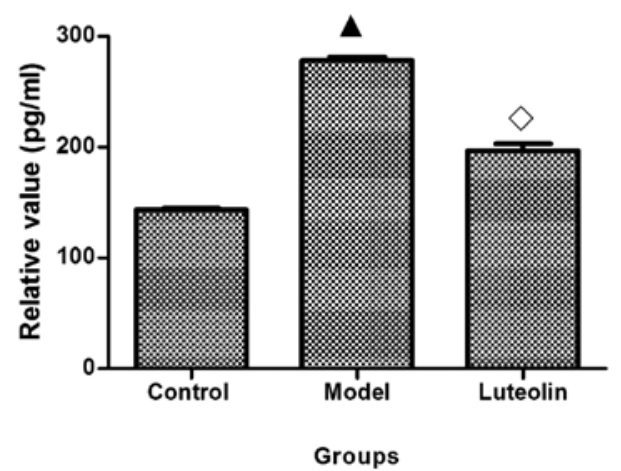

Serum TNF-a level

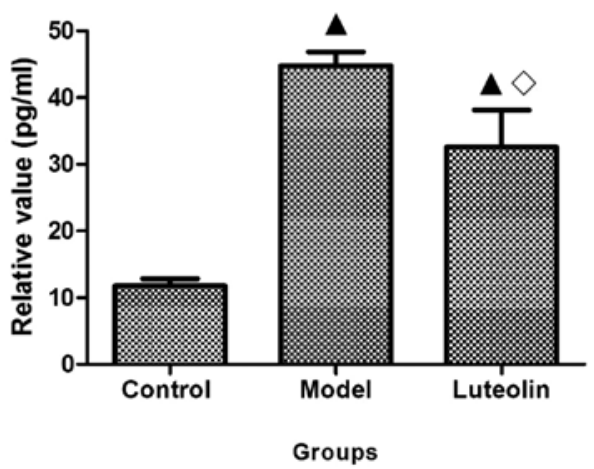

Figure 3. Effects of luteolin on TNF- $\alpha$ and IL-6. TNF- $\alpha$ and IL-6 levels in the sera of the three groups were detected by ELISA. Statistical significance vs. the control group $\left({ }^{\mathbf{\Delta}} \mathrm{P}<0.05\right)$; statistical significance vs. the model group $\left({ }^{\diamond} \mathrm{P}<0.05\right)$.

relationship between increased cytokines in serum and $\mathrm{NF}-\kappa \mathrm{B}$ signaling $(11,21)$, we speculated that luteolin exerts therapeutic effects by regulating NF- $\mathrm{B}$ signaling. Based on WB analysis, I $\mathrm{B}$ kinase $\beta$ (IKK $\beta$ ) and p-p65 were observed to be significantly upregulated in skeletal (Fig. 5A and B) and cardiac muscles (Fig. 5C and D) in the model mice, and the expression levels of these proteins were suppressed by luteolin.

In addition, proinflammatory cytokines can activate the NF- $\mathrm{B}$ pathway and p38 MAPK. For this reason, we assayed the levels of p38 and phospho (p)-p38. The levels of p38 were approximately equivalent in all three groups in the skeletal muscle (Fig. 5A and B) and in the cardiac muscle (Fig. 5C and D); however, there were obvious differences in the levels of p-p38. There was significant upregulation of p-p38 in both skeletal muscle and cardiac muscle in the model mice (Fig. 5A-D), which was inhibited by luteolin treatment in the skeletal muscle, while being unaffected by luteolin treatment in the cardiac muscle. 
A

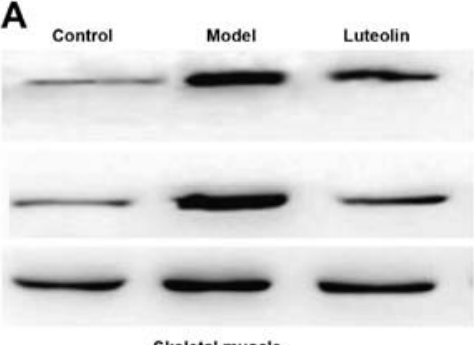

Skeletal muscle

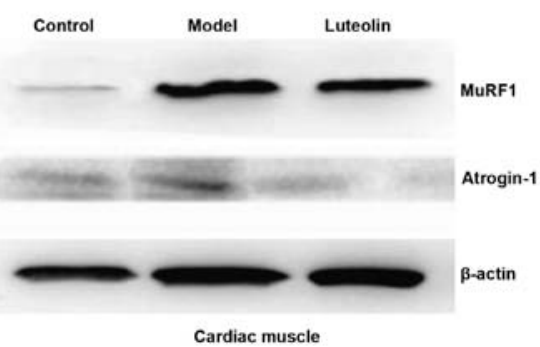

B $\quad \begin{gathered}\text { MuRF1 expression } \\ \text { in skeletal muscle }\end{gathered}$
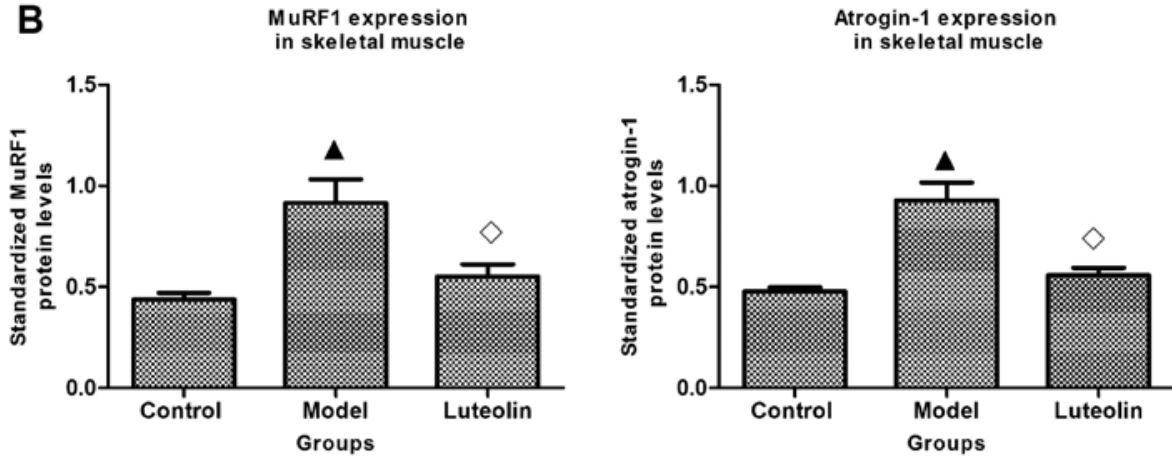

MuRF1 expression

in cardiac muscle
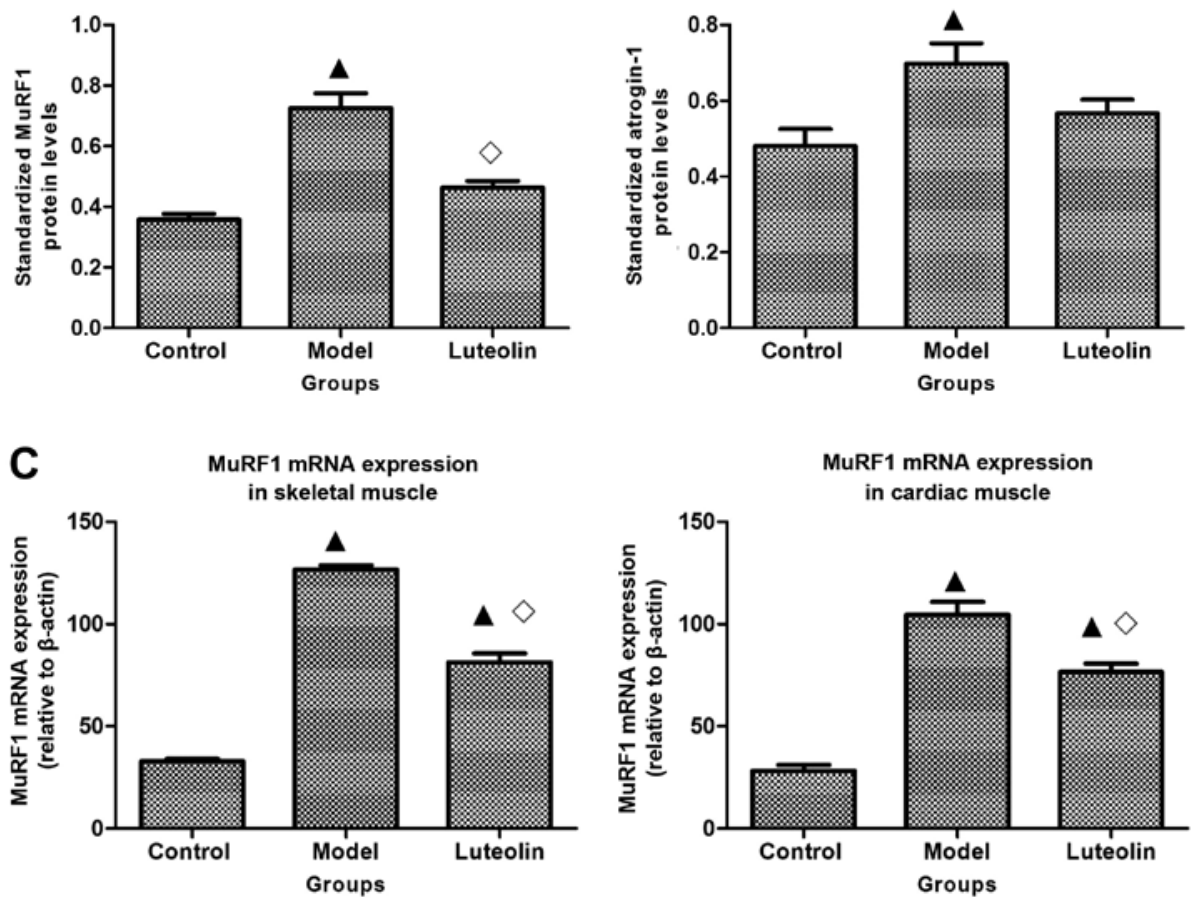

Atrogin-1 mRNA expression in skeletal muscle
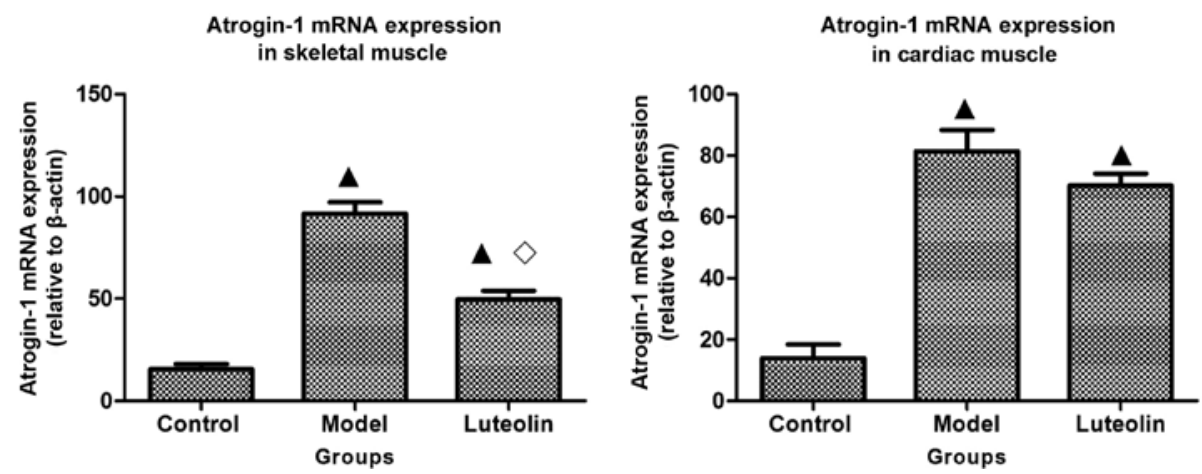

Figure 4. Effects of luteolin on atrogin-1 and MuRF1 expression. MuRF1 and atrogin-1 protein levels were detected by western blot (WB) analysis, and mRNA levels were assessed by qRT-PCR. (A) The luteolin group had lower MuRF1 expression in skeletal muscle and cardiac muscle and lower atrogin-1 expression in skeletal muscle. (B) The luteolin group showed significantly different band density for MuRF1 in skeletal muscle and cardiac muscle and significantly different atrogin-1 band density in skeletal muscle. (C) The luteolin group had significantly reduced MuRF1 mRNA expression in skeletal muscle and cardiac muscle and significantly reduced atrogin-1 mRNA expression in skeletal muscle. Statistical significance vs. the control group $\left({ }^{\boldsymbol{\Lambda}} \mathrm{P}<0.05\right)$; statistical significance vs. the model group $\left({ }^{\diamond} \mathrm{P}<0.05\right)$. 

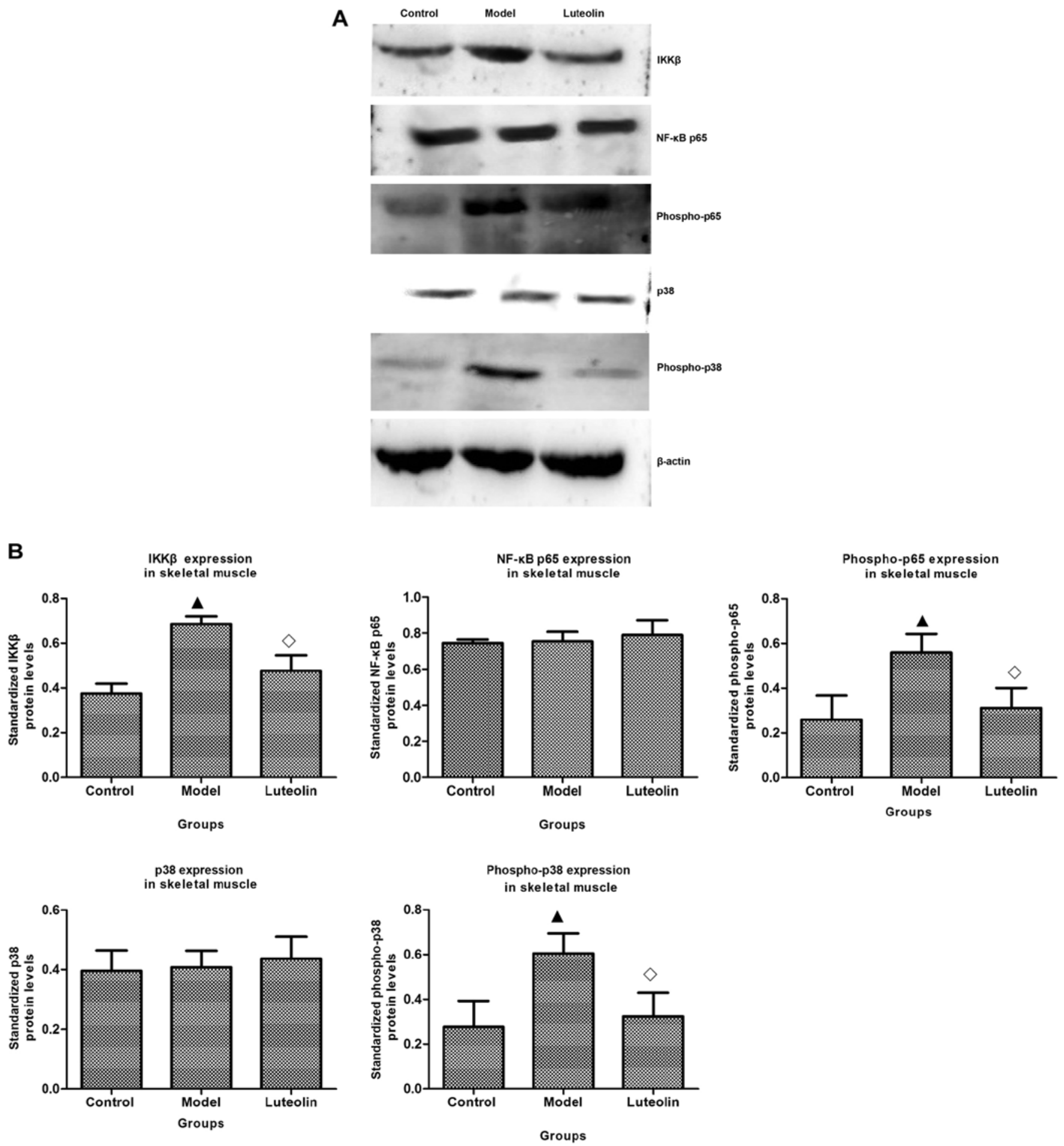

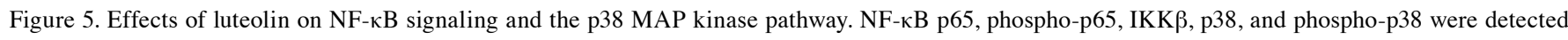
by western blot (WB) analysis as described in Materials and methods. (A) The luteolin group had lower phospho-p65, IKK $\beta$, and phospho-P38 expression in skeletal muscle and the same level of NF- $\mathrm{B}$, p65 and 38 expression compared with the model group. (B) The luteolin group had significantly different levels of phospho-p65, IKK $\beta$, and phospho-p38 in skeletal muscle, but exhibited no difference in NF- $\mathrm{B}$ p 65 and p38 expression compared with the model group.

\section{Discussion}

The primary characteristic of tumor-associated cachexia is muscle atrophy, and loss of skeletal muscle in cachexia results from decreased protein synthesis combined with increased protein degradation. The expression of the E3 ligase E3a-II is also reported to be significantly induced at the onset and during the progression of muscle wasting $(20,22)$. Meanwhile, E3a-II has been shown to be induced in myotubes by treatment with TNF- $\alpha$ or IL-6 $(17,23)$. Other studies confirm the importance of the IKK $\beta / \mathrm{NFKB}$ pathway in the induction of the ubiquitin-proteasome pathway (21).

Compounds of natural origin could be used as novel, innovative therapeutic agents for the treatment of cancer. Previous 

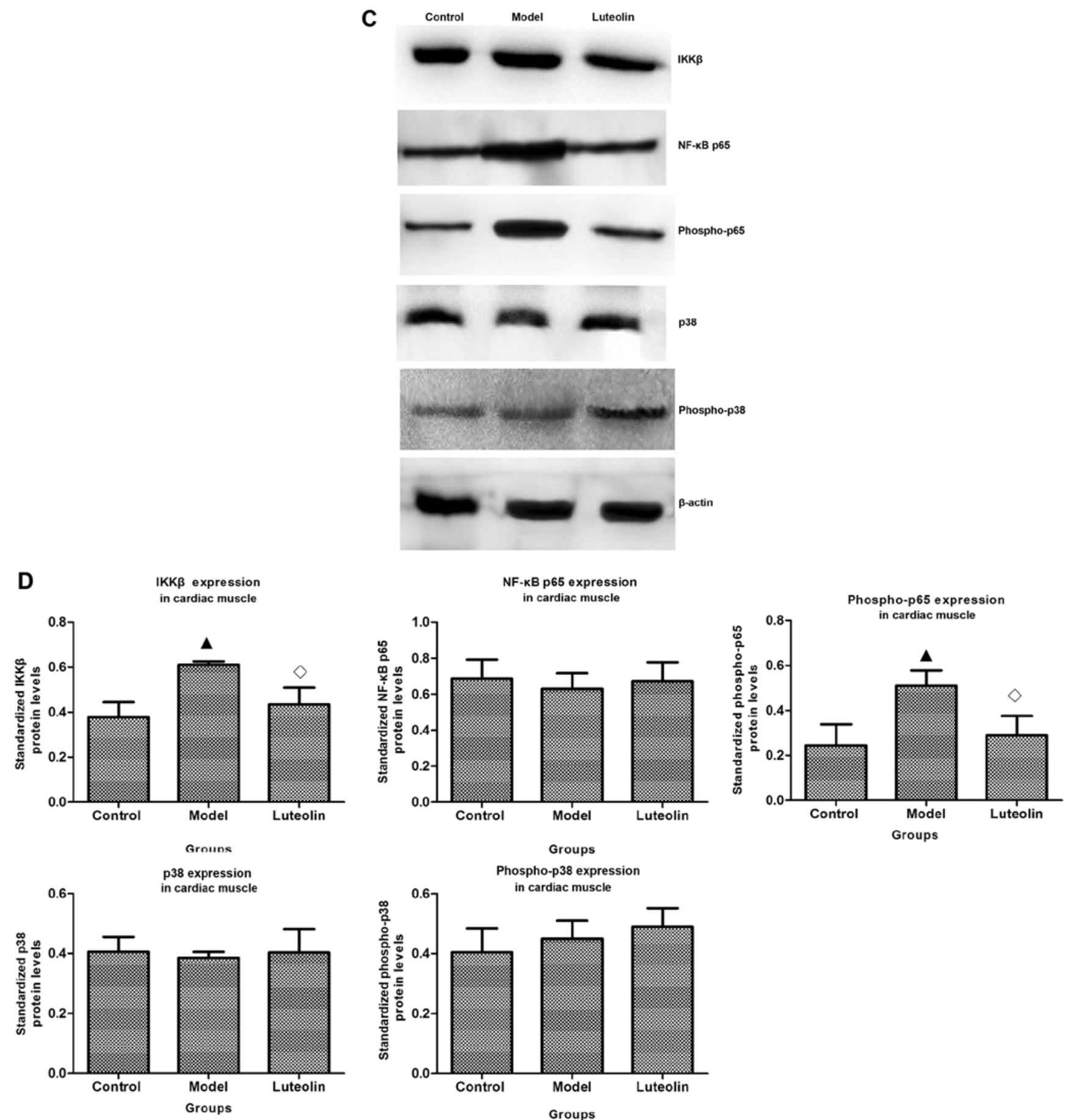

Figure 5. Continued. (C) The luteolin group had lower phospho-p65 and IKK $\beta$ expression in cardiac muscle compared with that in model mice, while the expression of NF- $\mathrm{kB}$ p65, p38, and phospho-p38 was similar between the model and luteolin groups. (D) The luteolin group had significantly different levels of phospho-p65 and IKK $\beta$ in cardiac muscle, but exhibited no difference in NF- $\mathrm{KB}$ p65, p38, and phospho-p38 expression compared with the model group. Statistical significance vs. the control group $\left({ }^{\boldsymbol{\Delta}} \mathrm{P}<0.05\right)$; statistical significance vs. the model group $\left({ }^{\diamond} \mathrm{P}<0.05\right)$.

studies have reported that a large number of phytochemical compounds may represent new anticancer compounds. For example, ginsenoside Rg3 has significant cancer-inhibitory activity; its suggested mechanisms of action include the induction of apoptosis, the inhibition of proliferation, metastasis and angiogenesis, and the promotion of immunity (24). As another example, we also reported that baicalin alleviated anorexia and inhibit skeletal muscle atrophy in experimental models of cancer cachexia (25). No effective treatments currently exist for the clinical treatment of cancer cachexia. Thus, inadequacies remain in the clinical management of cachexia due to the complex nature of the condition. However, as pathways continue to be identified, there is increased potential for more effective treatment of muscle wasting in cancer patients.

In the present study, we successfully replicated a cachexia model by subcutaneously injecting LLW cells into C57BL/6 mice according to procedures outlined in the literature (14-16). As muscle loss is the main characteristic of cachexia, which 
leads to insufficient muscle function, we first observed variation in muscle weight. Significant reductions in the gastrocnemius and heart muscle masses were observed in the tumor-bearing group. Such muscle mass loss was diminished in tumor-bearing mice that were administered luteolin; however, their muscle mass remained lower than that in the normal group. Moreover, the key markers of degradation in muscle, MuRF1 and atrogin-1, were upregulated in tumor-bearing mice, whereas this was suppressed by luteolin treatment.

In many cancers, TNF- $\alpha$ plays a paramount role in activating the $\mathrm{NF} \kappa \mathrm{B}$ pathway to induce a signaling cascade that promotes the progression of tumorigenesis. We measured the level of TNF- $\alpha$ in mouse serum, and the results confirmed increased TNF- $\alpha$ expression in the model group. However, TNF- $\alpha$ was restored to control group levels by luteolin intervention.

In addition, IL- 6 has been reported to have a regulatory role in muscle wasting during cachexia (23); however, an increased IL-6 level is not the most important activator of NF- $\kappa \mathrm{B}$. In this study, IL-6 levels were high in the model mice, and were reduced by luteolin treatment; however, the difference was not significant.

The purpose of the present study was to determine whether luteolin functions by targeting $\mathrm{NF}-\kappa \mathrm{B}$ signaling proteins and $\mathrm{NF}-\kappa \mathrm{B}$ transcription factors, which function in cancer-induced muscle wasting. The existing literature indicates the involvement of the $\mathrm{NF}-\kappa \mathrm{B}$ signaling protein, $\mathrm{I} \kappa \mathrm{B} \alpha$, in LLC tumor-bearing mice, and the overexpression of a super-repressor form of $\mathrm{I} \kappa \mathrm{B} \alpha$ in skeletal muscle was found to be associated with a $50 \%$ inhibition of muscle fiber atrophy (26). We detected the expression levels of MuRF1 and atrogin-1 in skeletal tissue, and observed that their levels were decreased by luteolin treatment, as compared with those in tumor-bearing mice administered vehicle treatment. The inhibition of classical NF- $\kappa \mathrm{B}$ signaling is sufficient to significantly decrease tumor-induced muscle loss, at least in mice, in part by inhibiting the upregulation of MuRF1. As one component of the complex, it is generally accepted that the activation of $\mathrm{NF}-\kappa \mathrm{B}$ requires the activation of either IKK $\alpha$ or $\operatorname{IKK} \beta(27)$; and the activation of IKK $\beta$ has been reported to cause profound muscle wasting that resembles clinical cachexia (21). For this reason, we also detected the expression of IKK $\beta$, and identified that its expression matched that of NF- $\kappa \mathrm{B}$.

It has been reported that MuRF1 activity is necessary to induce cardiac atrophy, and the significant dexamethasone-induced atrophy induced in the heart tissues of wild-type mice is essentially absent in $\mathrm{MuRF}^{-/}$mice (28). In addition, the present study observed that, in heart muscle tissue, the expression of MuRF-1 was upregulated in model mice, whereas this upregulation was attenuated in tumor-bearing mice administered luteolin. Therefore, we speculate that luteolin can inhibit the expression of MuRF1 through inhibition of classical NF- $\kappa \mathrm{B}$ signaling based on the current findings.

Although the expression of atrogin-1 in skeletal muscle tissue was also downregulated by luteolin treatment, its expression in heart tissue was the same as that in the model mice. Proinflammatory cytokines, such as TNF- $\alpha$, TWEAK and IL-1, stimulate two established pathways: the NF- $\kappa \mathrm{B}$ and the p38 MAPK pathways (29). Correspondingly, the expression levels of p38 and p-p38 were detected in skeletal muscle and heart muscle, and the results indicated that atrogin-1 expression was closely correlated with p38, which regulated partly the atrogin-1 expression. Moreover, MuRF1 and atrogin-1 are also regulated by other transcription factors and pathways (30-32); therefore, it is possible that luteolin reduces MuRF1 and atrogin-1 by mechanisms other than influencing $\mathrm{NF}-\kappa \mathrm{B}$ signaling in skeletal and heart muscle. Nonetheless, the current study indicated that luteolin limits the loss of cardiac and skeletal muscle and acts by inhibiting NF- $\kappa \mathrm{B}$ activation.

In conclusion, the present results clearly indicated that the natural flavonoid luteolin inhibited the production of the inflammatory mediators TNF- $\alpha$ and IL- 6 in a cancer cachexia model. To the best of our knowledge, this study is the first to report that luteolin inhibits the expression of MuRF1 by decreasing NF- $\kappa \mathrm{B}$ activation at both the transcriptional and translational levels. In addition, luteolin may alleviate the effects of atrogin-1 upregulation by reducing the expression of p38. Therefore, luteolin has the potential to be developed as a safe and effective alternative therapy for the treatment of cancer cachexia.

\section{Acknowledgements}

We acknowledge Dr Feng Shi (College of Pharmacy, Jiangsu University) for assisting in the assessment of this project at the beginning of the experiment.

\section{Funding}

The present study was supported by the Liaoning Natural Science Foundation (Grant no. 2015010571-301).

\section{Availability of data and materials}

The datasets used during the present study are available from the corresponding author upon reasonable request.

\section{Authors' contributions}

BL conceived and designed the experiment. BL, TC and YX performed the experiments. YJ analyzed the data. SM contributed the reagents/materials/analysis tools. BL and YW wrote the paper. All authors agreed to be accountable for all aspects of the work in ensuring that questions related to the accuracy or integrity of any part of the work are appropriately investigated and resolved.

\section{Ethics approval and consent to participate}

Animal research protocols carried out in this study were in accordance with the regulations of the Institutional Animal Care and Use Committee (IACUC) of Jiangsu University.

\section{Consent for publication}

Not applicable.

\section{Competing interests}

The authors declare that no competing interests exist. 


\section{References}

1. Fearon K, Strasser F, Anker SD, Bosaeus I, Bruera E, Fainsinger RL, Jatoi A, Loprinzi C, MacDonald N, Mantovani G,et al: Definition and classifi cation of cancer cachexia: An international consensus. Lancet Oncol 12: 489-495, 2011.

2. Argilés JM, Busquets S, Stemmler B and López-Soriano FJ: Cancer cachexia: Understanding the molecular basis. Nat Rey Cancer 14: 754-762, 2014

3. Evans WJ, Morley JE, Argilés J, Bales C, Baracos V, Guttridge D, Jatoi A, Kalantar-Zadeh K, Lochs H, Mantovani G, et al: Cachexia: A new definition. Clin Nutr 27: 793-799, 2008.

4. Bilir C, Engin H, Can M, Temi YB and Demirtas D: The prognostic role of inflammation and hormones in patients with metastatic cancer with cachexia. Med Oncol 32: 56, 2015.

5. Vaughan VC, Martin P and Lewandowski PA: Cancer cachexia: Impact, mechanisms and emerging treatments. J Cachexia Sarcopenia Muscle 4: 95-100, 2013.

6. Tisdale MJ: Mechanisms of cancer cachexia. Physiol Rev 89 381-410, 2009.

7. Fearon KC, Glass DJ and Guttridge DC: Cancer cachexia: Mediators, signaling, and metabolic pathways. Cell Metab 16 : 153-166, 2012.

8. Schcolnik-Cabrera A, Chávez-Blanco A, Domínguez-Gómez G and Dueñas-González A: Understanding tumor anabolism and patient catabolism in cancer-associated cachexia. Am J Cancer Res 7: 1107-1135, 2017.

9. Li H, Malhotra S and Kumar A: Nuclear factor-kappa B signaling in skeletal muscle atrophy. J Mol Med 86: 1113-1126, 2008.

10. Rhoads MG, Kandarian SC, Pacelli F, Doglietto GB and Bossola M: Expression of NF-kappaB and IkappaB proteins in skeletal muscle of gastric cancer patients. Eur J Cancer 46: 191-197, 2010.

11. Wysong A, Couch M, Shadfar S, Li L, Rodriguez JE, Asher S, Yin X, Gore M, Baldwin A, Patterson C, et al: NF- $\kappa \mathrm{B}$ inhibition protects against tumor-induced cardiac atrophy in vivo. Am J Pathol 178: 1059-1068, 2011.

12. Tuorkey MJ: Molecular targets of luteolin in cancer. Eur J Cancer Prev 25: 65-76, 2016.

13. Weng Z, Patel AB, Vasiadi M, Therianou A and Theoharides TC: Luteolin inhibits human keratinocyte activation and decreases $\mathrm{NF}-\kappa \mathrm{B}$ induction that is increased in psoriatic skin. PLoS One 9 e90739, 2014.

14. Merriman RL, Shackelford KA, Tanzer LR, Campbell JB, Bemis KG and Matsumoto K: Drug treatments for metastasis of the Lewis lung carcinoma: Lack of correlation between inhibition of lung metastasis and survival. Cancer Res 49: 4509-4516, 1989.

15. Au ED, Desai AP, Koniaris LG and Zimmers TA: The MEK-inhibitor selumetinib attenuates tumor growth and reduces IL-6 expression but does not protect against muscle wasting in Lewis lung cancer cachexia. Front Physiol 7: 682, 2017.

16. Chen X, Wu Y, Yang T, Wei M, Wang Y, Deng X, Shen C, Li W, Zhang H, Xu W, et al: Salidroside alleviates cachexia symptoms in mouse models of cancer cachexia via activating mTOR signalling. J Cachexia Sarcopenia Muscle 7: 225-232, 2016.
17. Todorov P, Cariuk P, McDevitt T, Coles B, Fearon K and Tisdale M: Characterization of a cancer cachectic factor. Nature 379: 739-742, 1996.

18. Albrecht JT and Canada TW: Cachexia and anorexia in malignancy. Hematol Oncol Clin North Am 10: 791-800, 1996.

19. Mueller TC, Bachmann J, Prokopchuk O, Friess H and Martignoni ME: Molecular pathways leading to loss of skeletal muscle mass in cancer cachexia - can findings from animal models be translated to humans? BMC Cancer 16: 75, 2016.

20. Bodine SC, Latres E, Baumhueter S, Lai VK, Nunez L, Clarke BA, Poueymirou WT, Panaro FJ, Na E, Dharmarajan K, et al: Identification of ubiquitin ligases required for skeletal muscle atrophy. Science 294: 1704-1708, 2001.

21. Cai D, Frantz JD, Tawa NE Jr, Melendez PA, Oh BC, Lidov HG, Hasselgren PO, Frontera WR, Lee J, Glass DJ and Shoelson SE: IKKbeta/NF-kappaB activation causes severe muscle wasting in mice. Cell 119: 285-298, 2004.

22. Clarke BA, Drujan D, Willis MS, Murphy LO, Corpina RA, Burova E, Rakhilin SV, Stitt TN, Patterson C, Latres E and Glass DJ: The E3 Ligase MuRF1 degrades myosin heavy chain protein in dexamethasone-treated skeletal muscle. Cell Metab 6: 376-385, 2007

23. Carson JA and Baltgalvis KA: Interleukin 6 as a key regulator of muscle mass during cachexia. Exerc Sport Sci Rev 38: 168-176, 2010.

24. Sun M, Ye Y, Xiao L, Duan X, Zhang Y and Zhang H: Anticancer effects of ginsenoside Rg3 (Review). Int J Mol Med 39: 507-518, 2017.

25. Li B, Wan L, Li Y, Yu Q, Chen P, Gan R, Yang Q, Han Y and Guo C: Baicalin, a component of Scutellaria baicalensis, alleviates anorexia and inhibits skeletal muscle atrophy in experimental cancer cachexia. Tumour Biol 35: 12415-12425, 2014.

26. Van Gammeren D, Damrauer JS, Jackman RW and Kandarian SC: The IkappaB kinases IKKalpha and IKKbeta are necessary and sufficient for skeletal muscle atrophy. FASEB J 23: 362-370, 2009.

27. Hayden MS and Ghosh S: Shared principles in NF-kappaB signaling. Cell 132: 344-362, 2008.

28. Willis MS, Rojas M, Li L, Selzman CH, Tang RH, Stansfield WE, Rodriguez JE, Glass DJ and Patterson C: Muscle ring finger 1 mediates cardiac atrophy in vivo. Am J Physiol Heart Circ Physiol 296: H997-H1006, 2009.

29. Egerman MA and Glass DJ: Signaling pathways controlling skeletal muscle mass. Cric Rev Biochem Mol Biol 49: 59-68, 2014.

30. Waddell DS, Baehr LM, van den Brandt J, Johnsen SA, Reichardt HM, Furlow JD and Bodine SC: The glucocorticoid receptor and FOXO1 synergistically activate the skeletal muscle atrophy-associated MuRF1 gene. Am J Physiol Endocrinol Metab 295: E785-E797, 2008

31. Stitt TN, Drujan D, Clarke BA, Panaro F, Timofeyva Y, Kline WO, Gonzalez M, Yancopoulos GD and Glass DJ: The IGF-1/PI3K/Akt pathway prevents expression of muscle atrophy-induced ubiquitin ligases by inhibiting FOXO transcription factors. Mol Cell 14: 395-403, 2004.

32. Sacheck JM, Ohtsuka A, McLary SC and Goldberg AL: IGF-I stimulates muscle growth by suppressing protein breakdown and expression of atrophy-related ubiquitin ligases, atrogin-1 and MuRF1. Am J Physiol Endocrinol Metab 287: E591-E601, 2004. 